\title{
Pasteless electrode for clinical use
}

\author{
C. J. De Luca \\ R. S. Le Fever \\ F. B. Stulen
}

Depanment of Orthopsedic Surgery. Children's Hospital Medical Center. Hervard Medical School. Boston, MA O2115. USA end Massachusetts Institute of Technology. Cambridge. MA. USA

\begin{abstract}
The design and construction of a clinically useful electrode for detecting the myoelectric signal is described. The requirements of the design were determined by a set of properties defined by clinicians and research engineers experienced in myoelectric signal detection. A novel, and seemingly useful. feature of this electrode is that the stainless-steel cans of two junction field-effect transistors are used as the recording surface while the same j.f.e.t.s operatt as the front end of a differential preamplifier. The electrode requires no paste or gel. In addition to its use as a stationary unit, it may be used as a probe to quickly and conveniently explore the myoelectric signals from many muscles.
\end{abstract}

Keywords-Electrodes. Myoelectric signa/s

\section{Introduction}

THE recent revitalisation of interest in mycelectric biofeedback and electrophysiological kinesiology, accompanied by the current upsurge in the quantitative assessment of muscle re-education exercises. has produced a need for an easy to use and reliable electrode for detecting the e.m.g. Or the myoelectric signal. Such an electrode would be convenient in a clinical environment.

In the past, various types of surface electrodes have been used to detect myoelectric signals. They can be conveniently grouped into three categories. The first category consists of a simple metal disc or plate in contact with the skin surface via a conductive paste or gel. Any metals with good conductivity may be used; the most common, however. are stainless steel and nickel. The lack of chemical equilibrium at the metal/electrolyte and electrolyte/skin junctions sets up a polarisation potential that may vary with temperature fluctuations. sweat accumulation, changes in the electrolyte concentration of the paste or gel. relative movement of the metal and skin, as well as the current flow: Various construction designs have been implemented in an attempt to stabilise the polarisation potential (BOTER et al., 1966; GIrTon and KamiYA, 1974).

The second category consists of the widely used silver/silver-chloride electrode, which also makes contact with the skin via a conductive paste or gel. This electrode has the advantage of having a reduced as well as more stable polarisation potential. Both of the above categories of electrodes work best if the skin is prepared by rubbing it with solvents to remove the oils and by treating it with abrasives to remove dead epidermis (Shackel, 1959; TAM

Firs received 14 th August and in final form 11th October 1978

$0140-0118 / 79 / 030387+04 \$ 01 \cdot 50 / 0$

(6) IFMBE : 1979

Medical \& Biological Engineering \& Computing and Webster, 1977: BLrbalik and Webster, 1978). This procedure reduces the impedance of the skin/ electrolyte interface.

A third category of electrodes has been employed to eliminate the need for skin preparation and conducting medium. These have been referred to as the dry electrodes. These electrodes can be either resistively coupled (LEWES. 1965; BERUEY el al.. 1971) or capacitively coupled to the skin (LOPEZ and Richardson, 1969: POTTER and ME.TKe, 1970; BETTS and BROU:, 1976). In the case of the capacitively coupled electrode. the recording surface is coated with a thin layer of dielectric substance and the skin electrode junction behaves as a capacitor. Although the capacitively coupled electrodes have the advantage of not requiring a conductive medium, they have a higher inherent noise level (POTTER and MENKE, 1970). Also, these electrodes do not have a long term reliability because their dielectric properties are susceptible to change with the presence of perspiration and the erosion of the dielectric substance.

The design presented in this paper represents an improvement to the current forms of resistively coupled electrodes.

\section{Electrode requirements}

Inquiries made of clinicians and researchers experienced in the use of myoelectric-signal electrodes indicated that a suitable electrode unit (including its associated electronics) should have the following desirable properties:

\section{Physical properties}

(a) it should be easily and quickly affixed to and removed from the skin of an individual 
(b) it should not require a conductive paste or gel between the electrode surface and the skin

(c) it should be small in size, light in weight and ruggedly constructed

(d) it should not be cumbersome or obtrusive to either the subject or the clinician

(e) it should be inexpensive and easy to construct.

\section{Elecirical properties}

(a) it should be a surface differential electrode whose associated electronics should have a common mode to differential conversion ratio of at least $5000(74 \mathrm{~dB})$ at $60 \mathrm{~Hz}$

(b) it should have an actual input impedance of at least $10^{8} \Omega$ at $100 \mathrm{~Hz}$ : note that the input resistance will always be shunted by some inherent or stray capacitance. which will reduce the actual impedance

(c) the d.c. input bias current should be kept at a minimum, thereby reducing the polarisation potential

(d) the a.c. output impedance should be sufficiently low (less than $2 \mathrm{k} \Omega$ ) to reduce any signa attenuation, common mode inbalance caused by the output-cable capacitance, $60 \mathrm{~Hz}$ inductive pickup and motion artifacts generated in the output cable

(e) it should not restrict the bandwidth of the myoelectric-signal processor

$(f)$ it should be electrically stable so as not to have time dependent effects on the characteristics of the detected myoelectric signal

$(g)$ it should have a thermal noise less than $2 \mu \mathrm{V}$ r.m.s. over the banduidth of the recorded myoelectric signal (higher noise levels reduce the effectiveness of the electrode in detecting signals from weak muscies)

(h) it should have a stable, reliable and judiciously located ground reference contact. The location of the ground contacts with respect to the recording contacts must be a compromise: the

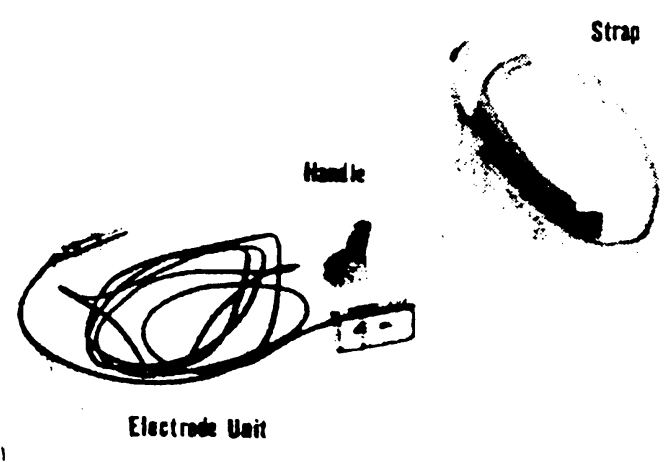

Fig. 1 Electrode unit. handle and strap distance should be sufficiently small to minimise the size of the electrode unit, but sufficiently large so that the recording contacts do not short to ground when the skin perspires

(i) it should have a low current drain.

\section{Design and construction}

The above requirements can be achieved by a suitably designed resistively-coupled dry electrode consisting of a pair of unity-gain buffer preamplifiers located very close to the recording surfaces. This is a well known strategy that has been used for the past decade (LOPEZ and RICHARDSON, 1969).

After attempting several configurations, the following design emerged. The electrode unit consists of four basic components: the metal shell. which performs a dual function as a housing for the electrode and as the ground contact: the attachments, consisting of a strap and a handle: the recording contacts and the cable. The complete unit is presented in Fig. 1.

The shell consists of a milled block of highly pure $(99 \cdot 71 \%)$ titanium metal (ASTM No. 8348). The external measurements are $30 \mathrm{~mm}$ long. $15 \mathrm{~mm}$ wide and $7 \mathrm{~mm}$ deep. Metal is removed from the interior of the block by milling, forming a rectangular shell having a wall thickness of $2 \mathrm{~mm}$. Titanium metal of high purity is used because it is highly resistant to oxidation, has outstanding corrosion resistance, can be milled with relative ease and is relatively inexpensive. However, any corrosiveresistant conductive metal can be used. The rectangular border of the shell acts as the ground contact and the remainder of the shell forms a shield against the inevitable ambient electromagnetic radiation. A hooked Velcro strap cemented to the back of the shell mates with the attachments that support the electrode.

The recording surfaces are formed by the cans of the two junction field-effect transistors (j.f.e.t.s). which have a diameter of $4.5 \mathrm{~mm}$. Their cans, made of stainless steel, are polished. The j.f.e.t.s (2N4220A) are used as both the recording surfaces and the front end of the two buffer preamplifiers. The two j.f.e.t.s are cemented with epoxy glue in the middle of the shell. Their centres are spaced $10 \mathrm{~mm}$ apart. This spacing allows the electrode unit to be as small as possible while maintaining sufficient separation so that the recording surfaces will not short when the skin perspires. The tops of the cans protrude $1 \mathrm{~mm}$ above the surface of the border of the electrode shell. This protrusion is essential to insure that the recording surfaces will always touch the skin. The weight of the electrode unit, excluding the cable, is $5 \cdot 2 \mathrm{~g}$.

The lead from each of the j.f.e.t. cans (pin 4) is soldered to its corresponding gate lead (pin 3 ). This arrangement reduces the stray capacitance in the front end, which in turn increases the actual 
input impedance. The j.f.e.t.s act as unity gain buffers in a constant-current source follower arrangement, show'n schematically in Fig. 2. The drain leads (pins 1) of the f.e.t.s, are tied together and supplied with a positive voltage (typically $+4-5 \mathrm{~V}$ ) through

ELCCTROOE CABLE IMSTRUMENTATION
UKIT
AMPLIFIER

for the j.f.e.t. sources and set the bias current through the j.f.e.t.s (typically $100 \mu \mathrm{A}$ ).

With this arrangement, instrumentation amplifiers with relatively large d.c. input bias currents $(<100 \mu \mathrm{A})$ may be used, such as an Analog Devices

\section{CURRENT SINKS}

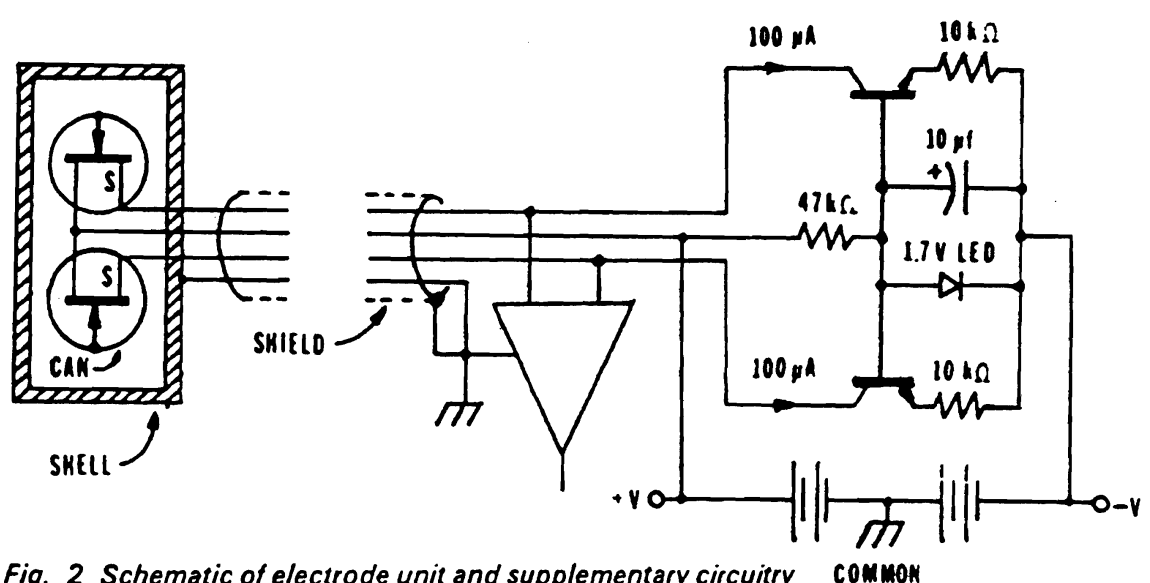

Fig. 2 Schematic of electrode unit and supplementary circuitry common

one lead in a four conductor shielded cable. The cable shield and another conductor connect the circuit ground (common) to the titanium shell of the electrode to provide the ground reference. The source leads (pins 2) of the j.f.e.t.s are connected through the two other leads in the shielded cable to the differential inputs of an instrumentation amplifier. This amplifier should have a high a.c. input impedance $(>100 \mathrm{M} \Omega$ ). The two $n-p-n$ transistors located next to this amplifier act as constant current sinks 521. The instrumentation amplifier provides all of

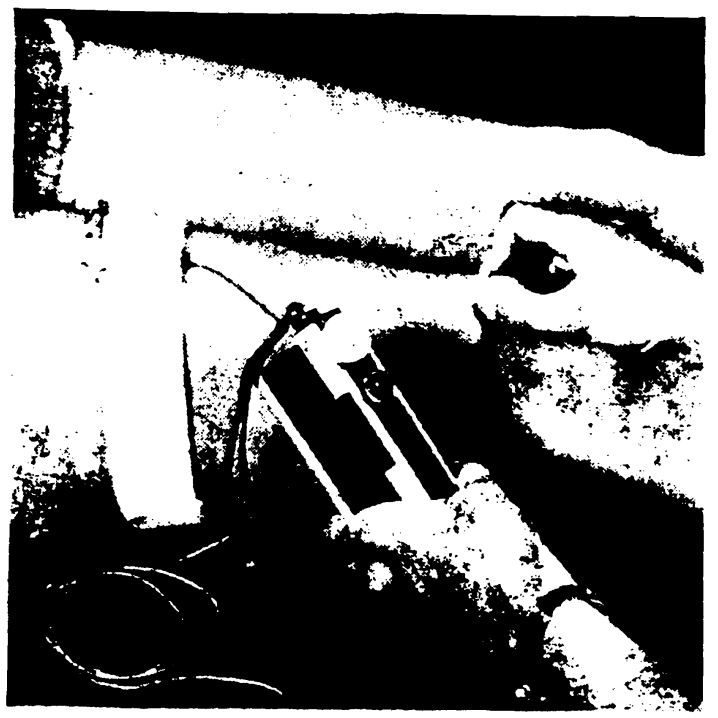

Fig. 3 Electrode unit secured to limb with strap implement the common mode rejection, anplification and bandwidth limitation of the electrode unit. The instrumentation amplifier and its associated electronics, as well as the current sink circuit, are physically separated from the electrode unit by the cable, hence, they can conveniently reside in the device to which the electrode is attached. This arrangement reduces the cost of the electrode unit when it becomes necessary to replace it.

The described configuration of each buffer preamplifier of the electrode unit has the typical performance characteristics shown in Table 1.

Table 1. Typical performance characteristics

\begin{tabular}{|c|c|}
\hline Bandwidth & d.c. to $>100 \mathrm{kHz}$ \\
\hline C. input bias current & $<100 \mathrm{pA}$ \\
\hline C. input impedance & $\begin{array}{c}>10^{10} \Omega \text { paralleled by } \\
5 \mathrm{pF}\end{array}$ \\
\hline C. output impedance & $1.6 \mathrm{k} \Omega$ \\
\hline $\begin{array}{l}\text { loise (differential) } \\
\text { inputs grounded } \\
\text { each input with } 10 \mathrm{k} \Omega \text { to } \\
\text { ground }\end{array}$ & $\begin{aligned}< & 0.04 \mu \mathrm{V} \text { r.m.s. } / \sqrt{ } \mathrm{Hz} \\
< & 0.05 \mu \mathrm{V} \text { r.m.s. } / \sqrt{ } \mathrm{Hz} \\
(<0.7 \mu \mathrm{V} \text { r.m.s. for } & 0.700 \mathrm{~Hz} \text { bandwidth })\end{aligned}$ \\
\hline $\begin{array}{l}\text { Common mode to differential } \\
\text { conversion ratio } \\
\text { (similar to c.m.r.r.) }\end{array}$ & $\begin{array}{l}>75 \mathrm{~dB} \text { at } 60 \mathrm{~Hz} \\
\text { (driving } 500 \mathrm{pF} \text { to } \\
\text { ground of cable } \\
\text { capacitance) }\end{array}$ \\
\hline ower supply drain & $\sim 350 \mu \mathrm{A}$ at $\pm 4.5 \mathrm{~V}$ \\
\hline
\end{tabular}

Two devices for affixing the electrode to the skin have been developed: one is a strap and the other is 
a handle. The strap consists of two strips of Velcro pile sewn back to back with a piece of elastic nylon webbing sewn to each end. The free end of one of the elastic strips is attached to two small strips of Velcro hooks that are sewn back to back. The end of the other elastic strip is sewn to a loop of Velcro pile that loops through a $D$ ring. This arrangement allous the strip to encircle body segments of varying perimeters. The handle consists of a plastic rod with a Velcro patch cemented to one end. This also mates with the Velcro patch on the back of the electrode shell. These implements can be seen in Fig. 1.

When the electrode unit is to be stationed over a particular muscle, the unit is affixed to the Velcto pile on the strap via mating Velcro hooks, and the strap is urapped around a body segment. Typically. the electrode unit is positioned over a limb muscle. as shown in Fig. 3. However, with a longer strap the electrode unit can be attached to the thorax. The strap arrangement assures that the electrode unit is anchored with sufficient pressure to make a stable contact with the skin. The elastic strips in the strap allow it to expand when the muscle is contracted, thereby eliminating the uncomfortable pressure sensation and restricted blood flow that would otherwise be experienced by the subject. No paste or gel is required. If the skin making contact with the electrode unit is clean. the electrode assembly will become electrically stable within 5 to $10 \mathrm{~s}$. Otherwise, the unit should be removed and the skin should be swabbed with rubbing alcohol. which should be allowed to evaporate prior to re-attaching the electrode unit.

Alternately, the electrode unit may be used as a probe by attaching the handle to it. In this configura-

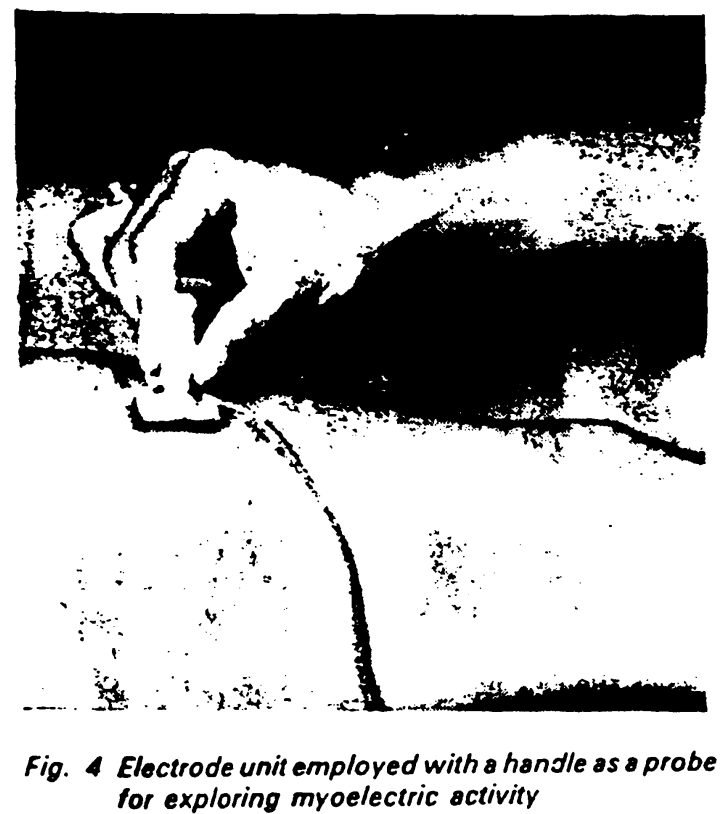

tion the electrode unit is simply held, with some pressure, against the skin over the muscle of interest. as shown in Fig. 4. To guarantee consistent and reliable performance, it is preferable to rub the electrode face on the skin area on which it is to be located.

The electrode unit may serve as the front end for most apparatus used for recording the myoelectric signal. Currently, the electrode unit is used with a myofeedback device constructed in our laboratory (see Fig. 3). The myofeedback devices have been used on a regular basis by physical therapists. The convenience and effective performance of the electrode unit were well received in a busy, clinical environment. They found the probe feature and freedom from the need for a paste or gel particularly. convenient. No problems with the electrode unit were reported during eight months of daily use in clinics.

1cinon:ledgment-We are grateful for the clinical infut provided by C. McCarthy and the technical assistance of $\mathbf{L}$. D. Gilmore and P. Nott. This project was supported in part by RSA Grant No. 23-P-55854 of DHEW.

\section{References}

Bergey, G. E., Squires, R. D. and Sipple. W. C. (1971) Electrocardiogram recording with pasteless electrodes. IEEE Trans., BME-18, 206.

BetTs. R. P. and Brown, B. H. (1976) Method for recording electrocardiograms with dry electrodes applied to unprepared skin. Med. \& Biol. Eng.. 14. 313-315.

Boter. J., Den Hertog, A. anj Kuiper, J. (1966) Disturbance-free skin electrodes for persons during exercise. Med. \& Biol. Eng., 4, 91.

Bltbank, D. P. and Webster. J. G. (1978) Reducing skin potential motion artefact by skin abrasion. Med. \& Biol. Eng., 16, 31-38.

Girton, D. G. and KamiYa. J. (1974) A very stable electrode system for recording human scalp potentials with direct-coupled amplifiers. Eleciroenceph. Clin. Neurophysiol., 37, 85.

LeuEs, D. (1965) Multipoint electrocardiography without skin preparation, Lancel, 3rd July, 17.

Lopez, A. Jun., and Richardson, P. C. (1969) Capacitive eloctrocardiographic and bioelectric electrodes. IEEE Trans., BME-16, 99.

PotTER, A. and MENKE, L. (1970) Capacitive type of biomedical electrode. IEEE Trans., BME-17, 350.

SHACKEL, B. (1959) Skin drilling: a method of diminishing galvanic skin potentials. Am. J. Psych., 72, 114.

TAM, H. and Webster, J. G. (1977) Minimizing electrode motion artifact by skin abrasion. IEEE Trans.. ME-24, 134. 